\title{
Cryo-EM structural analysis of the SARS-CoV-2 Nucleocapsid protein
}

Michael Casasanta ${ }^{1}$, G.M. Jonaid ${ }^{2}$, Liam Kaylor ${ }^{2}$, William Luqiu ${ }^{3}$, Maria Solares ${ }^{2}$, Mariah Schroen ${ }^{4}$, William Dearnaley ${ }^{5}$, Jarad Wilson ${ }^{6}$, Madelin Dukes ${ }^{7}$ and Deborah Kelly ${ }^{2}$

${ }^{1}$ Pennsylvania State University, State College, Pennsylvania, United States, ${ }^{2}$ Pennsylvania State University, University Park, Pennsylvania, United States, ${ }^{3}$ Duke University, United States, ${ }^{4}$ Pennsylvania State University, United States, ${ }^{5}$ Pennsylvania State University, University park, Pennsylvania, United States, ${ }^{6}$ RayBiotech, United States, ${ }^{7}$ Protochips, United States

Cryo-electron microscopy (cryo-EM) is an optimal technique to study a large swathe of biological samples ranging from whole cells to individual proteins (Carroni \& Saibil, 2016; Zhang et al., 2017; Kang et al., 2018; Wrapp et al., 2020). We recently published a highly reproducible microchip-based approach to study protein complexes from human cancer cells that help mitigate some of these difficulties. This methodology allowed us to elucidate protein structures in the molecular weight range of $\sim 100 \mathrm{kDa}$ (Varano et al., 2019; Alden et al., 2019). Here we present an improved approach with redesigned microchips which have been optimized to visualize low-molecular weight proteins for cryo-EM analysis. In our case, we used the SARS-CoV-2 Nucleocapsid $(\mathrm{N})$ protein $(\sim 48 \mathrm{kDa})$ as our model protein. We chose the SARS-CoV-2 $\mathrm{N}$ protein because of its essential role in virus survival and for its therapeutic targeting potential (Zeng et al., 2020; McBride et al., 2014). The N protein also elicits a high immune response in those infected and is often utilized as the prime target in commercially available SARS-CoV-2 antibody-based detection kits (Diao et al., 2021; Li et al., 2020; Woo et al., 2004).

The $\mathrm{N}$ protein was expressed and purified from bacteria, and it is commercially available from RayBiotech, Inc. To prepare cryo-EM specimens, we employed Ni-NTA-coated microwell-integrated microchips (Protochips, Inc) with imaging windows with the dimensions of $10 \mu \mathrm{m} \times 10 \mu \mathrm{m}$ in the $\mathrm{x}$ - and $\mathrm{y}$ - dimension and $\sim 20 \mathrm{~nm}$ in thickness. Aliquots of His-tagged $\mathrm{N}$ protein were added to the microchips then flash-frozen in liquid ethane. Specimens were imaged using a Talos F200C Transmission Electron Microscope (TEM) (ThermoFisher Scientific). Using our microchip-based approach we determined the first complete structure of the $\mathrm{N}$ protein to $4.5 \AA$ which revealed features of the flexible $\mathrm{N}$-terminal motif and the helical-rich C-terminal region (Figure 1a,b). The structure showed a distinct "top hat" motif in the first 50-amino acids of the N-protein, which is predicted to lack secondary elements. We posit that the N-terminal His-tag of the recombinant protein served to tether the protein to the coated microchips, providing stability. Additionally, there is sufficient density to accommodate the helix comprised of residues D216-N228 as well as many of the adjacent side chains in this region (Figure 1c). There are also strong features in the $\mathrm{C}$-terminal aspect of the protein. As such, we can visualize multiple rigid helices present in this region which served as antibody $(\mathrm{Ab})$ binding site in plasma samples from COVID-19 patients. Using a standard model for the Fab structure (pdb 4QXG), we narrowed down the Ab attachment site to a flexible loop region including the residues Q384-A397 (Peng et al., 2015)(Figure 1d). This loop appears to be highly flexible and surface exposed. Overall, these data provide new structural information for the SARS-CoV-2 N protein with and without human Abs, presenting a prime opportunity to determine other small protein structures as future pandemics arise in society. 


\section{SARS-CoV-2 Nucleocapsid protein}
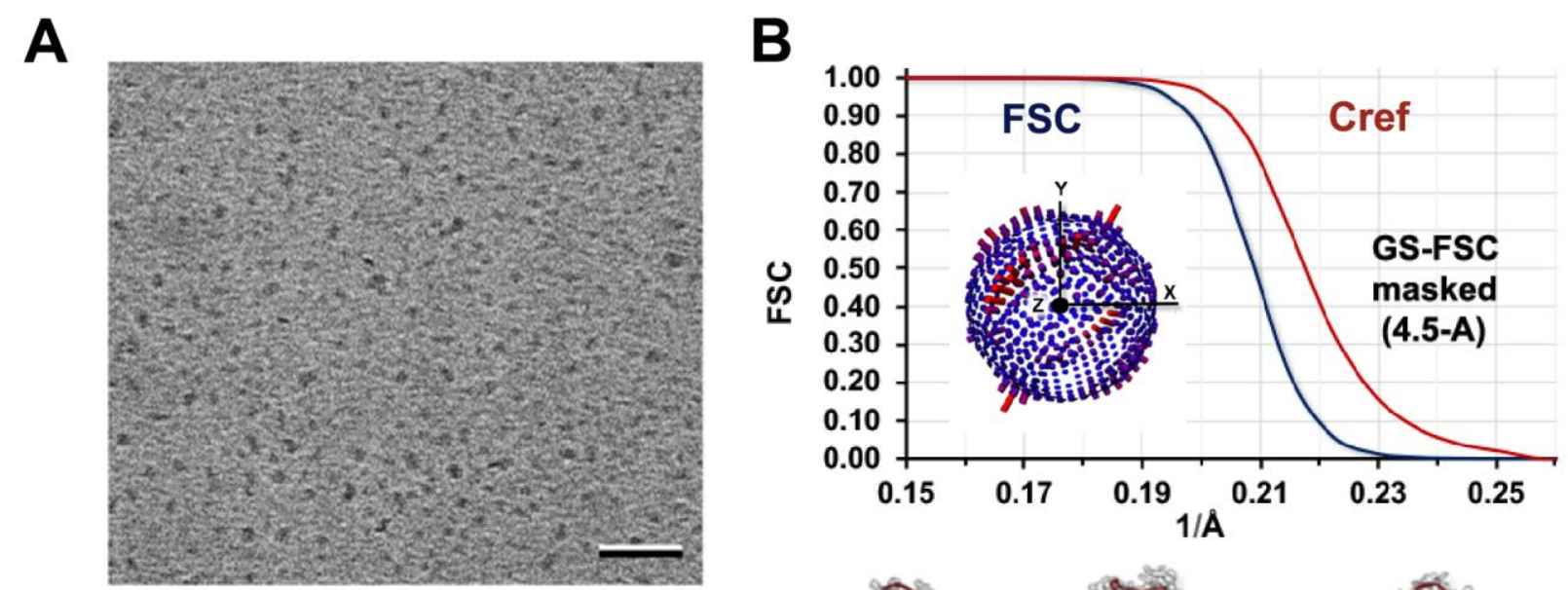

C
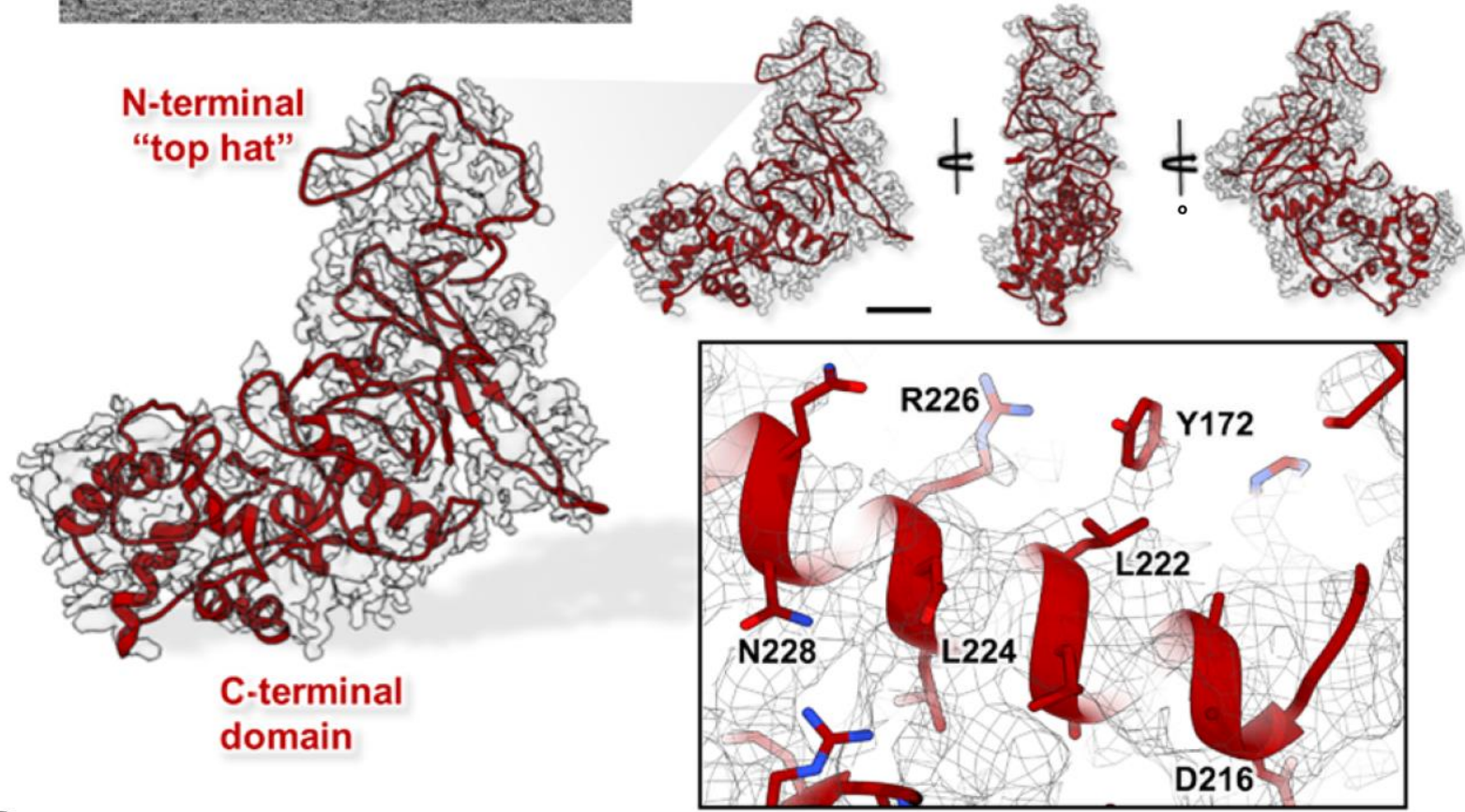

D

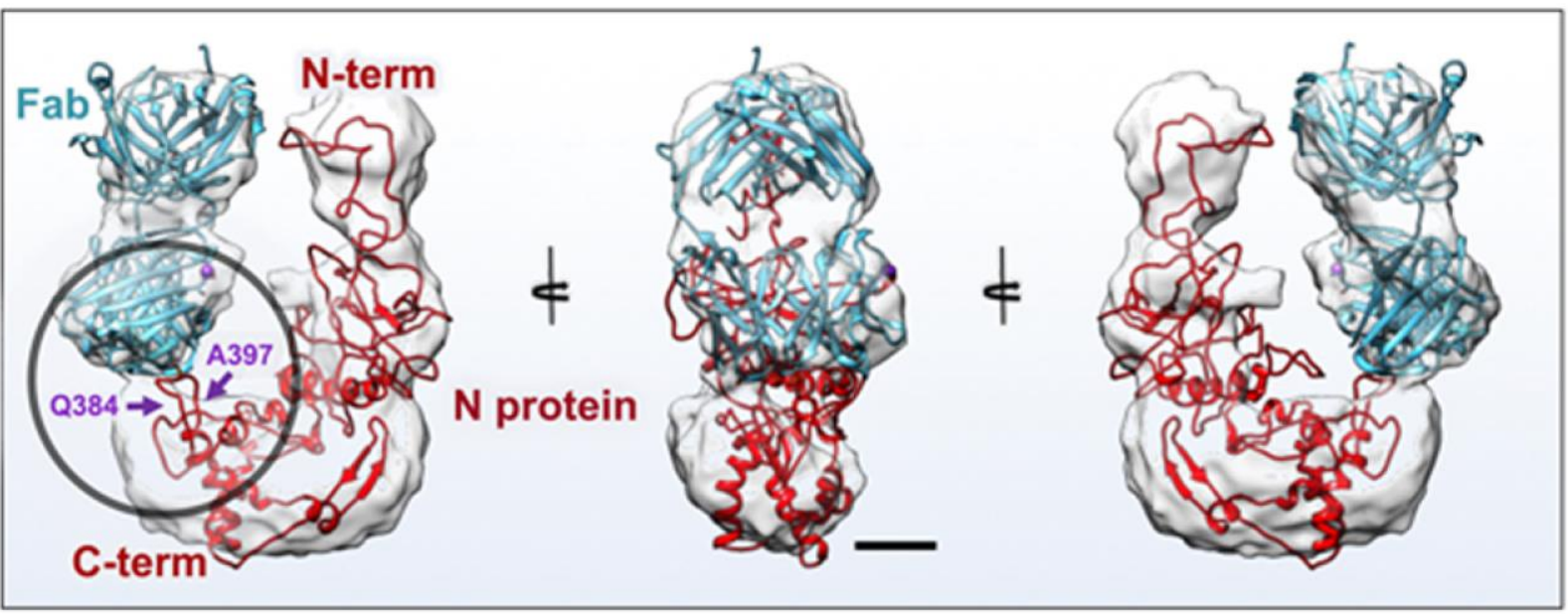


Figure 1. Figure 1. Structural insights of the SARS-CoV-2 N protein with and without antibody attachment. (A). Representative micrograph of frozen hydrated specimens. Scale bar is $20 \mathrm{~nm}$. (B). The angular distribution plot shows the particle orientation lacks major limitations. The Fourier Shell Correlation and Cref (0.5) evaluation indicate a spatial resolution of $4.5 \AA$ at the 0.143 value. (C). The Cryo-EM density map of the $\mathrm{N}$ protein shows distinct structural features at both the $\mathrm{N}$ and $\mathrm{C}$-terminus of the protein. Rotational views highlight the model fit within the density. The inset shows the central helix in the structure from residues D216 to N228 defined a boundary between the two domains. Scale bar is $10 \AA$ A. (D). Cryo-EM map resolved to 14.2- $\AA$ shows the of the $\mathrm{N}$ protein (red) and a model for a Fab fragment (cyan). A flexible loop comprised of residues Q384 - A397 is proximal to the Fab-binding site. Rotational views of the map provide visual clarity of the physical relationship between the two models. Scale bar is $10 \AA$.

\section{References}

Alden, N. A., Varano, et al. (2019). Cryo-EM-On-a-Chip: Custom-Designed Substrates for the 3D Analysis of Macromolecules. Small 15,

Cameron Varano, et al. (2019). Customizable Cryo-EM Chips Improve 3D Analysis of Macromolecules. Microscopy and microanalysis 25, 1310-1311.

Carroni, M. \& Saibil, H. R. (2016). Cryo electron microscopy to determine the structure of macromolecular complexes. Methods 95, 78-85.

Diao, B.,et al. (2021). Accuracy of a nucleocapsid protein antigen rapid test in the diagnosis of SARSCoV-2 infection. Clinical microbiology and infection: the official publication of the Euro. Soc. of Clin. Micro. and Infectious Diseases 27, 289.e1-289.e4.

Kang, Y., et al. (2018). Cryo-EM structure of human rhodopsin bound to an inhibitory G protein. Nature 558, 553-558.

Li, T.,et al. (2020). Serum SARS-COV-2 Nucleocapsid Protein: A Sensitivity and Specificity Early Diagnostic Marker for SARS-COV-2 Infection. Front. in cellular and infection micro. 10, 470.

McBride, R., van Zyl, M. \& Fielding, B. C. (2014). The coronavirus nucleocapsid is a multifunctional protein. Viruses 6, 2991-3018.

Peng, L., Oganesyan, V., Wu, H., Dall'Acqua, W. F. \& Damschroder, M. M. (2015). Molecular basis for antagonistic activity of anifrolumab, an anti-interferon- $\alpha$ receptor 1 antibody. $m A b s$ 7, 428-439.

Woo, P. et al. (2004). Longitudinal profile of immunoglobulin G (IgG), IgM, and IgA antibodies against the severe acute respiratory syndrome (SARS) coronavirus nucleocapsid protein in patients with pneumonia due to the SARS coronavirus. Clin. and diagnostic lab. immunology 11, 665-668.

Wrapp, D., et al. (2020). Cryo-EM structure of the 2019-nCoV spike in the prefusion conformation. Science 367, 1260-1263.

Zeng, W., et al. (2020). Biochemical characterization of SARS-CoV-2 nucleocapsid protein. Biochem. and biophys. research comm. 527, 618-623.

Zhang, Y., et al. (2017). Cryo-EM structure of the activated GLP-1 receptor in complex with a G protein. Nature 546, 248-253. 